\title{
Curvature and Singularity Driven Diffusion for Oriented Pattern Enhancement with Singular Points *
}

\author{
Qijun Zhao, Lei Zhang, David Zhang, Wenyi Huang, and Jian Bai \\ Biometrics Research Centre, Department of Computing \\ The Hong Kong Polytechnic University, Hong Kong \\ \{csqjzhao, cslzhang, csdzhang, cswyhuang, csjbai\}@comp.polyu.edu.hk
}

\begin{abstract}
Oriented patterns, e.g. fingerprints, consist of smoothly varying flow-like patterns, together with important singular points (i.e. cores and deltas) where the orientation changes abruptly. Gabor filters and anisotropic diffusion methods have been widely used to enhance oriented patterns. However, none of them can well cope with regions of varying curvatures or regions surrounding singular points. By incorporating the ridge curvatures and the singularities into the diffusion model, we propose a new diffusion method to better exploit the global characteristics of oriented patterns. Specifically, we first locate the singular points, and regularize the estimated orientation field by using a singularity driven nonlinear diffusion process. We then enhance the oriented patterns by applying an oriented diffusion process which is driven by the curvature and singularity. Experiments on synthetic data and real fingerprint images validated that the proposed method is capable of consistently enhancing oriented patterns while well preserving the ridge structures in singular regions.
\end{abstract}

\section{Introduction}

Oriented patterns, also known as oriented or flow-like textures, have been attracting much research attention in applications of computer vision $[4,17]$ and pattern recognition such as fingerprint recognition [13] for a long time. Referring to Fig. 1, fingerprint is a typical example of oriented patterns consisting of smoothly varying flow-like patterns (namely ridge structures), together with important singularities (namely cores and deltas) [7]. Oriented patterns are locally anisotropic with their ridge orientation field being

\footnotetext{
* The work is partially supported by the CERG fund from the HKSAR Government, the central fund from Hong Kong Polytechnic University, the Natural Science Foundation of China (NSFC) under Contract No. 60620160097, and the National High-Tech Research and Development Plan of China (863) under Contract No. 2006AA01Z193 and 2007AA01Z195.
}

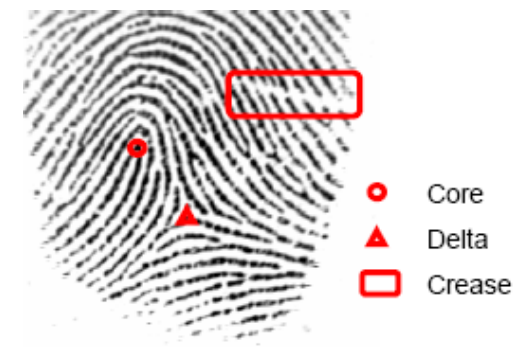

Figure 1. Fingerprint, a typical example of oriented patterns.

well defined except at singular points [4]. Singular points, viewed as important global features of oriented patterns, are the points of discontinuity of the orientation field $[4,7,13]$. Two kinds of singularities are widely used in literature as shown in Fig. 1: the core is the end point of the innermost curving ridge, and the delta is the confluence point of three different flow directions [7, 13]. The singular regions surrounding singular points have the highest ridge curvatures across the oriented patterns, and the orientation around the singular points changes abruptly.

For real images of oriented patterns, the image quality can be degraded significantly by a number of factors, including the corrupted noise in the image acquisition process, the creases and other interference that would break the ridges (referring to Fig. 1). The enhancement of oriented patterns is thus to reduce the noise and interference to make the pattern structures easier for recognition. It is well known that traditional linear and isotropic smoothing methods such as Gaussian smoothing will blur or destroy ridge structures [10]. Therefore, some nonlinear and anisotropic smoothing methods were developed later $[8,18,11,15,2,20]$.

A popular approach to oriented pattern enhancement is to use contextual filters that are tuned to proper orientations and frequencies [8, 18, 11]. For instance, Hong et al. [11] proposed to use a bank of Gabor filters to enhance the oriented patterns on fingerprint images. They calculated the dominant ridge orientation and the average ridge frequency 
at each pixel based on a neighborhood to the pixel. A Gabor filter was then applied, which is tuned to the local ridge orientation and frequency at the pixel. This method, if the Gabor filters are tuned to correct orientations and frequencies, can successfully suppress the noise on the ridges and remove the creases. However, such contextual filter based methods have three shortcomings. First, there is often block effect on the enhanced oriented pattern images because the ridge frequency is usually estimated block by block. Second, when there are singular points on the ridge patterns, it will be very difficult to accurately estimate the frequency around the singular points. As a result, the contextual filters can not accurately enhance the ridge patterns, but instead often destroy the ridge structures in these regions. Third, when the images are of very poor quality, it will be hard to calculate the ridge frequency. Consequently, the contextual filters will fail to effectively enhance the oriented patterns.

As a kind of partial differential equation (PDE) based methods, various diffusion techniques [15, 2, 20] have been proposed in the community of computer vision and image processing in the past decades. They are proven to be very useful for image de-noising, restoration, enhancement, and feature extraction [15, 2, 20, 16]. Some nonlinear and anisotropic diffusion methods have been recently applied to oriented pattern processing, including ridge orientation estimation [14,5] and ridge pattern enhancement $[1,6,9,21,5,10]$. In general, these methods first estimate the ridge orientation field on the oriented pattern images and then steer intensity diffusion processes primarily along the ridge orientation. Advantages of the nonlinear and anisotropic diffusion-based methods include that they are free from frequency estimation and that they can better preserve ridge edges.

However, most existing nonlinear and anisotropic diffusion methods consider neither the local ridge curvatures nor the global singular points of the oriented patterns when determining the diffusivity. Consequently, they have two drawbacks. First, they use similar diffusivity at locations where the ridges could have different curvatures. In fact, when the ridges have small curvatures, we can apply a large amount of diffusion on the ridges. On the contrary, when the ridges bend sharply, the diffusion should be reduced. By taking into consideration the local ridge curvatures, we can make the diffusion more consistently. Second, the existing nonlinear and anisotropic diffusion methods ignore the singular points by simply applying the same diffusion process in both singular regions and non-singular regions. This may destroy the ridge structures in the singular regions due to the rapid change of ridge orientation around singular points.

The goal of this paper is to explicitly incorporate the local ridge curvatures and the global singular point features into the diffusion model such that it can not only consistently enhance the oriented patterns in non-singular regions, but also well preserve the ridge structures in singular regions. To this end, we first estimate an initial ridge orientation field and locate the singular points on the oriented pattern, and then regularize the ridge orientation field by using a nonlinear diffusion process which is driven by the singularity of oriented patterns. Consequently, a curvature and singularity driven diffusion process is applied to enhance the ridge pattern.

The rest of the paper is organized as follows. Section 2 presents the proposed curvature and singularity driven diffusion model. Section 3 presents the enhancement algorithm. Section 4 shows experiments on synthetic data and typical real fingerprint images. Section 5 concludes the paper.

\section{The curvature and singularity driven diffu- sion model}

The basic idea of diffusion is to exchange quantities between adjacent objects, and as the diffusion goes the whole system will evolve to a smoother and smoother status [19]. In the context of image processing, the exchanged quantities are the intensities of pixels in the image. Mathematically, the diffusion process in an image $I(x, y)$ can be formulated as the following PDE

$$
\partial_{t} u=\operatorname{div}(D \cdot \nabla u)
$$

with initial condition

$$
u(x, y ; 0)=I(x, y)
$$

Here, $\nabla u=\left(u_{x}, u_{y}\right)^{T}$ is the gradient vector (' $T$ ' denotes the transpose) and $D$ is the diffusivity term, which can be either a scalar or a $2 \times 2$ matrix.

When $D$ is a constant scalar, the diffusion equation (1) defines a linear isotropic diffusion, which will blur the edges on the image. To better preserve the edges, Perona and Malik [15] proposed to define the diffusivity by a decrease function of the local gradient magnitudes as follows

$$
D=g\left(|\nabla u|^{2}\right)=\left(1+|\nabla u|^{2} / k^{2}\right)^{-1}
$$

where $k$ is a parameter. By using this diffusivity, the diffusion will be reduced at those locations which have a larger likelihood to be edges. Although the above model can adapt the diffusivity to the local features, at each pixel it still diffuses identically in all directions. Therefore, it does not have the ability of orientation selectivity.

Later, Weickert [19] proposed to use a diffusion tensor to tune the diffusion directions according to the dominant orientation at each pixel. The diffusion tensor is defined as

$$
D=\left(p_{1} p_{2}\right)\left(\begin{array}{cc}
\lambda_{1} & 0 \\
0 & \lambda_{2}
\end{array}\right)\left(p_{1}^{T} p_{2}^{T}\right)
$$


where $\lambda_{1}$ and $\lambda_{2}$ are the diffusivities along the two orthogonal diffusion directions $p_{1}$ and $p_{2}$ respectively. In Weickert's diffusion model, $p_{1}$ and $p_{2}$ are defined by the two eigenvectors of the following local structure tensor (suppose $p_{1}$ corresponds to the larger eigenvalue)

$$
S_{\rho}=G_{\rho} *\left(\nabla u \nabla u^{T}\right)
$$

where $G_{\rho}$ is a Gaussian kernel with standard variance $\rho$, '*' denotes convolution, and the diffusivities along these two directions are determined by the two eigenvalues of $S_{\rho}$. Basically, we can steer the diffusion process along any orientation with any diffusivity by adjusting the four components in the diffusion tensor.

Our diffusion model for oriented pattern enhancement originates from Weickert's model. For oriented patterns, the ridge orientation can be approximately estimated before the diffusion. Therefore, it is not necessary to do the structure tensor analysis in each step of the diffusion, which is quite time-consuming. In addition, the oriented patterns consist of locally parallel dense patterns [4]. Thus, to enhance the oriented patterns, we should diffuse along the ridge orientation but never the perpendicular orientation. Let $\theta$ be the ridge orientation at a pixel. The diffusion orientation $p_{1}$ at the pixel is then defined as

$$
p_{1}=(\cos \theta, \sin \theta)^{T}
$$

The diffusivity along this orientation is positive $\left(\lambda_{1}>0\right)$, while the diffusivity along its perpendicular orientation is set to zero $\left(\lambda_{2}=0\right)$. Therefore, the diffusion is along $p_{1}$ only, i.e. the ridge orientation at the pixel.

As discussed in the introduction, the oriented patterns are globally characterized by singular points and the ridge curvatures change greatly between singular regions and nonsingular regions. It is important to take these characteristics into consideration when enhancing the oriented patterns. Bearing this in mind, we define the diffusivities as

$$
\begin{gathered}
\lambda_{1}=\exp \left\{-\left(\frac{c^{2}}{k_{1}^{2}}+\frac{1}{k_{2}^{2} \cdot d_{S}^{2}}\right)\right\} \\
\lambda_{2}=0
\end{gathered}
$$

Here, $k_{1}$ and $k_{2}$ are two parameters to control the diffusion velocities, $c$ is the ridge curvature at the pixel, and $d_{S}$ is the distance from the pixel to the closest singular point (if there is no singular point, $d_{S}$ is infinity). By substituting (4) and (6-8) into (1), we obtain the curvature and singularity driven oriented diffusion model as follows

$$
\partial_{t} u=u_{\theta \theta} \cdot \exp \left\{-\left(\frac{c^{2}}{k_{1}^{2}}+\frac{1}{k_{2}^{2} \cdot d_{S}^{2}}\right)\right\}
$$

where $u_{\theta \theta}=u_{x x} \cos ^{2} \theta+u_{y y} \sin ^{2} \theta+2 u_{x y} \cos \theta \sin \theta$.
In the proposed diffusion model, the diffusion is exactly along the ridge orientation. It diffuses fast in small curvature regions and non-singular regions and will slow down while it approaches to high curvature regions or singular points. Such adaptivity is desired in order to consistently enhance the oriented patterns in regions of different curvatures and preserve the ridge structures in singular regions.

\section{The enhancement algorithm}

In this section, we will develop a new algorithm for oriented pattern enhancement based on the proposed diffusion model in (9). We first introduce the method for singular point detection and orientation field regularization and then present the enhancement scheme.

\subsection{Singular point detection and orientation field regularization}

In order to use the curvature and singularity driven oriented diffusion model to enhance oriented patterns, we need to estimate the ridge orientation field and detect the singular points in the oriented patterns. Meanwhile, in order to suppress noise in the orientation field, we need to regularize the orientation field. However, regularization such as smoothing will lead to the shifting of singular points. Therefore, some tradeoff has to be made between regularizing the orientation field and preserving the singular points. In this section, we propose to regularize the orientation field by explicitly considering the singular points.

According to Perona's observation [14], the singular points of oriented patterns have the causal property: singular points may disappear, but not appear, when diffusing in the orientation field. Inspired by this finding, we propose to detect the singular points in the process of orientation diffusion. We start from an initial estimation of the ridge orientation field which is obtained by analyzing the local structure tensor as defined in (5) [3]. Because a large scale smoothing of the structure tensor will lead to large displacements of singular points, we use a small scale in the structure tensor analysis. Once we have the initial ridge orientation field (referring to Fig. 2(a)), we locate the singular points on it by using the Poincare index [13]. At this moment, we may find many singular points, some of which might be false ones (see Fig. 2(b)).

We then apply to the orientation field a linear diffusion process as defined in (1) by setting $D$ as a constant. Because the value of orientation is within the range of 0 to $\pi$, which is intrinsically discontinuous [14], we map the orientation $\theta$ to a continuous complex plane as

$$
U=\cos (2 \theta)+i \sin (2 \theta)=R+i I
$$

where $R=\cos (2 \theta)$ and $I=\sin (2 \theta)$ are the real and imaginary parts. The diffusion is then conducted on these two 


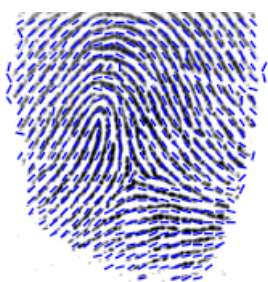

(a)

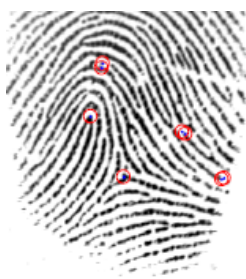

(b)

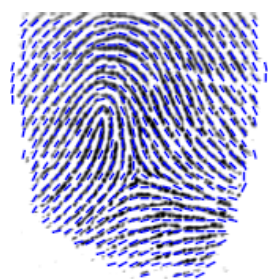

(c)

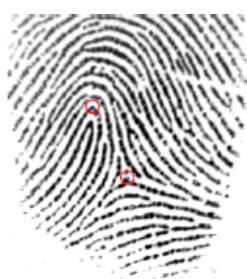

(d)

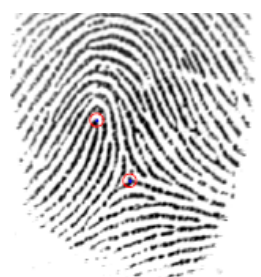

(e)

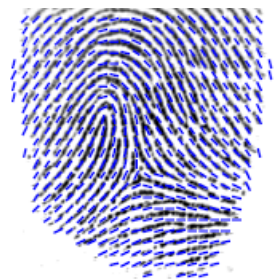

(f)

Figure 2. Singular point detection and orientation field regularization. (a) shows the initial orientation field, and (b) shows the singular points detected on it; (c) is the orientation field after linear diffusion of 50 iterations; (d) shows the singular points detected on the smoothed orientation field in (c); (e) shows the final singular point detection results; and (f) is the final orientation field regularized by using the singularity driven nonlinear diffusion.

parts simultaneously. After diffusion by $N$ iterations (in our experiments, $N=10$ ), we can retrieve a smoother orientation field from the diffused real and imaginary parts according to $\theta=\frac{1}{2} \arctan (I / R)$. We then detect the singular points on the obtained smoother orientation field. If the number of singular points keep unchanged for a sufficient number of iterations, or a specific number of iterations has been reached, the diffusion in the ridge orientation field stops. Otherwise, we diffuse for $N$ more iterations. Fig. 2(c) shows the smoothed orientation field after 50 iterations of diffusion.

When the diffusion stops, the false singular points will be eliminated but the survived singular points may shift from their true locations due to the smoothing effect of diffusion as can be seen in Fig. 2(d). For more accurate localization of these singular points, we turn to the detection results in the initial ridge orientation field. The singular points in the initial ridge orientation field which are the closest ones to those survived singular points are taken as the final singular point detection results. By using such a feedback scheme, we can not only remove false singular points, but also locate the true singular point more accurately (see Fig. 2(e)).

Based on the detected singular points, we can now regularize the initial orientation field. Here, we adopt a nonlinear diffusion process to reduce the diffusion around singular points such that the orientation field close to the singular points can be better preserved. To this end, we define the diffusivity at a pixel $(i, j)$ based on its distance to the closest singular points as follows

$$
D(i, j)=\exp \left\{-\frac{1}{k^{2} d_{S}^{2}(i, j)}\right\}
$$

where $k$ is a constant, and

$$
d_{S}(i, j)=\min \left\{d_{s}(i, j) \mid s=1,2, \cdots, n\right\}
$$

with $d_{s}$ being the distance between the pixel and the $s^{\text {th }}$ singular point, and $n$ is the total number of singular points. With the above defined singularity driven nonlinear diffusion on the orientation field, most noisy orientation can be corrected while preserving the orientation around singular points. Fig. 2(f) shows an example of the final orientation field regularized by the singularity driven nonlinear diffusion. Compared with the regularized orientation field in Fig. 2(c), the proposed method successfully avoids over smoothing the orientation field around singular points while correctly regularizing the orientation at the crease and other interference.

\subsection{Oriented pattern enhancement}

Once the ridge orientation field and the singular points have been obtained, we can now apply the curvature and singularity driven oriented diffusion model to enhance the oriented patterns. Different from conventional diffusion applications, for oriented pattern enhancement, we calculate only once the diffusivity at each pixel before the diffusion, and then use this diffusivity throughout the whole diffusion process. This makes our algorithm more efficient. Referring to the definition of the diffusion model in (9), the diffusion orientation $\theta$ is just the ridge orientation at the pixel, and the diffusivity will be determined by the local ridge curvature and its distance to the closest singular point. The distance at the pixel is calculated according to (12). As for the curvature, we refer to the orientation difference proposed by Perona [14]. Specifically, we calculate the curvature at the pixel $(i, j)$ as follows

$$
c(i, j)=\sum_{\left(i^{\prime}, j^{\prime}\right) \in N(i, j)}\left(1-\cos \left(2 \theta\left(i^{\prime}, j^{\prime}\right)-2 \theta(i, j)\right)\right)
$$

where $N(i, j)$ denotes the neighborhood to the pixel $(i, j)$. In our experiments, we use the 4-connected neighborhood. Finally, the diffused intensity at the pixel $(i, j)$ in the $(t+1)^{\text {th }}$ iteration can be calculated by

$$
\begin{aligned}
& u(i, j ; t+1)=u(i, j ; t)+ \\
& u_{\theta \theta}(i, j ; t) \cdot \exp \left\{-\left(\frac{c(i, j)^{2}}{k_{1}^{2}}+\frac{1}{k_{2}^{2} d_{S}^{2}(i, j)}\right)\right\}
\end{aligned}
$$




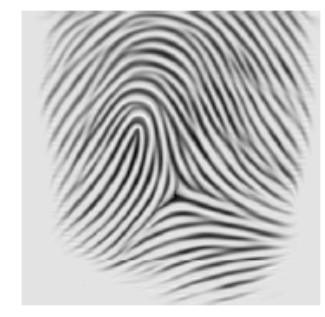

(a)

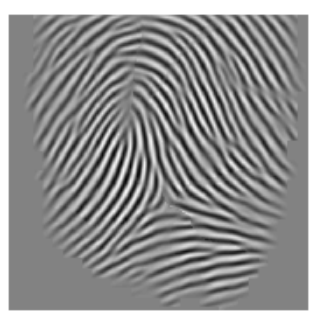

(b)
Figure 3. Enhancement results of the image in Fig. 1 by using (a) the proposed method and (b) the Gabor filters.

As the diffusion proceeds, noise as well as other distractions like creases on ridges and valleys will be gradually removed. On the resulting enhanced oriented patterns, broken ridges and valleys are connected and the ridges and valleys are becoming more separable. More importantly, the ridge structures in the singular regions are also well preserved. The parameters involved in the diffusion model and the number of iterations can be determined by experience for specific oriented pattern data according to their quality and to the application requirement.

Fig. 3 shows the enhancement results of Fig. 1 in comparison with the Gabor filter based enhancement method [11]. While the Gabor filter based method has obvious block effect especially in the singular regions, we see that the proposed method enhances much better the ridge structures in singular regions than the Gabor filters.

\section{Experimental results}

We first use a synthetic toy example to illustrate the effectiveness of including the ridge curvature in the diffusion. As shown in Fig. 4(a), in the synthetic oriented pattern image, there are some straight lines and some curves which have different curvatures. They are broken by a straight line, resulting in a break point on each line and each curve. We use the proposed curvature driven oriented diffusion method as well as the other two representative oriented diffusion methods $[6,10]$ to repair the breaks.

The oriented diffusion in [6] sets the diffusivity using a method similar to that of Weickert's model [20], which is based on local structure tensor analysis. In [10], the diffusivity is set to be a constant. The results of the three methods after 10 iterations and 20 iterations are shown in Figs. 4(b)-4(d) and Figs. 4(e)-4(g) respectively. As can be seen from the results, the curvature driven oriented diffusion enhances regions of different curvatures more consistently, whereas the diffusion driven by local structure tensor analysis [6] performs quite differently in flat and curved regions. Besides, compared with the other oriented diffusion methods [6, 10], the curvature driven oriented diffusion method can repair the curves more smoothly (note the intensity along the lines and curves). This is because neither the local structure tensor analysis [6] nor the constant diffusivity [10] can capture the curved characteristics of oriented patterns (note that the structure tensor uses the first order derivatives whereas the curvature is a second order derivative feature).

In order to assess the effect of singularity on enhancing oriented patterns, we use a variety of real fingerprint images for experiments. These images are taken from the FVC2002 fingerprint image databases [12]. In our experiments, we first use the histogram equalization to pre-process the fingerprint images such that the contrast between ridges and valleys is improved. We compare the proposed method with the representative Gabor filter based method [11] and the state-of-the-art oriented diffusion based methods [6, 10] according to their performance in enhancing various patterns with singular points. In the literature of fingerprint classification, according to the singular points on the fingerprints, there are generally six classes of oriented patterns, i.e. arch, tented arch, whorl, twin loop, left loop, and right loop [13]. We thus consider four cases in our experiments: one core point, one core point and one delta point, two core points on a whorl pattern, two core points on a twin loop pattern. Fig. 5 shows four typical example images used in the experiments.

These images are enhanced by the Gabor filter based method [11], Cheng's method [6], Hastings' method [10], and the proposed method (all the three diffusion based methods conduct 30 iterations of diffusion). The enhancement results are given in Fig. 6. In the results of Gabor filter based method, obvious block effect can be observed (especially in regions close to singular points) and the ridge edges are displaced. The diffusion based methods do not have these problems. However, as can be seen from the marked regions on the results, when there are singular points, the Gabor filter based method and the other two diffusion based methods fail to accurately enhance the ridge structures in the singular regions. On the contrary, the proposed method successfully preserves the ridge structures in the singular regions by well controlling the diffusion in these regions. Take the third row in Fig. 6 as an example. All the counterpart methods destroy the ridge structures in the singular regions, i.e. the innermost ridge is broken by them. But the proposed method preserves it very well. Overall, the proposed method not only enhances very well the ridge structures in non-singular regions, but also accurately preserves the ridge structures in singular regions.

\section{Conclusions}

This paper studied the problem of enhancing oriented patterns with singular points. Oriented patterns like fingerprints are commonly seen in computer vision and pattern recognition relevant applications such as biometrics. In 


\section{Wm(t)}

(a)

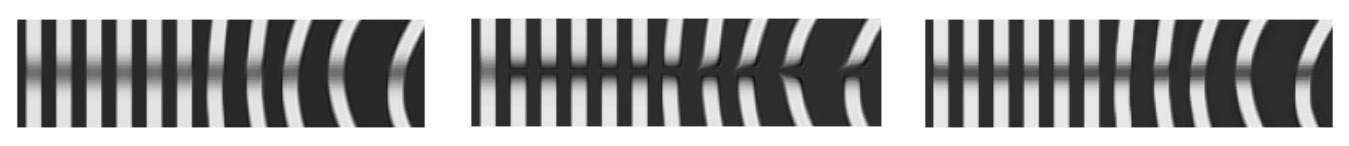

(b)

(c)

(d)

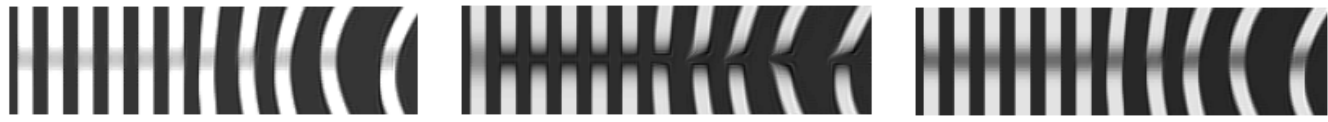

(e)

(f)

(g)

Figure 4. Toy example for curvature driven oriented diffusion. (a) is the original oriented pattern image. (b-d) are the enhancement results by the proposed method, Cheng's method [6], and Hastings' method [10], after 10 iterations, and (e-g) after 20 iterations.
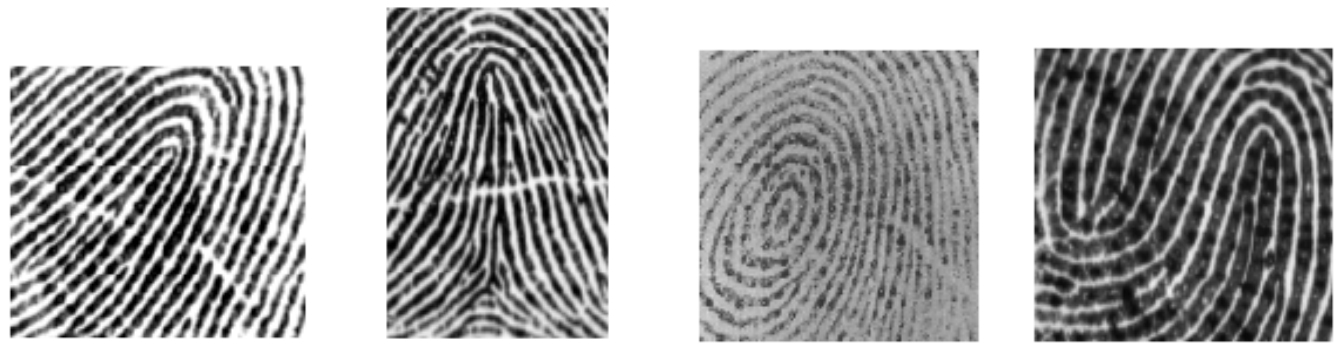

Figure 5. Four typical fingerprints with different singular points.

this paper, we proposed a novel oriented diffusion based approach. It inherits the merits from diffusion based enhancement methods, such as frequency estimation free and good edge preservation. Moreover, the proposed oriented diffusion model determines the diffusivity based on ridge curvatures and singular points. Our experiments on both synthetic data and real fingerprint images demonstrated that the proposed method could not only enhance the ridge structures in different regions more consistently, but also enhance the ridge structures in singular regions more accurately. Such improvement on oriented pattern image enhancement is expected to benefit the following feature extraction and pattern recognition processes.

\section{References}

[1] A. Almansa and T. Lindeberg. Fingerprint enhancement by shape adaptation of scale-space operators with automatic scale selection. IEEE Transactions on Image Processing, 9(12):2027-2042, 2000. 2

[2] L. Alvarez and L. Mazorra. Signal and image restoration using shock filters and anisotropic diffusion. SIAM Journal of Numerical Analysis, 31:590-605, 1994. 1, 2

[3] A. M. Bazen and S. H. Gerez. Systematic methods for the computation of the directional fields and singular points of fingerprints. IEEE Transactions on Pattern Analysis and Machine Intelligence, 24(7):905-919, 2002. 3

[4] O. Ben-Shahar and S. W. Zucker. The perceptual organization of texture flow: a contextual inference approach. IEEE Transactions on Pattern Analysis and Machine Intelligence, 25(4):401-417, 2003. 1, 3

[5] H. Chen and G. Dong. Fingerprint image enhancement by diffusion processes. ICIP2006, pages 297-300, 2006. 2

[6] J. Cheng, J. Tian, H. Chen, Q. Ren, and X. Yang. Fingerprint enhancement using oriented diffusion filter. AVBPA2003, pages 164-171, 2003. 2, 5, 6

[7] S. C. Dass. Markov random field models for directional field and singularity extraction in fingerprint images. IEEE Transactions on Image Processing, 13(10):1358-1367, 2004. 1

[8] L. O. Gorman and J. V. Nickerson. An approach to fingerprint filter design. Pattern Recognition, 22(1):29-38, 1989. 1

[9] Y. Hao and C. Yuan. Fingerprint image enhancement based on nonlinear anisotropic reverse diffusion equations. IEEE EMBS2004, pages 1601-1604, 2004. 2

[10] R. Hastings. Ridge enhancement in fingerprint images using oriented diffusion. Digital Image Computing Techniques and Applications, pages 245-252, 2007. 1, 2, 5, 6

[11] L. Hong, Y. Wan, and A. K. Jain. Fingerprint image enhancement: Algorithm and performance evaluation. IEEE 

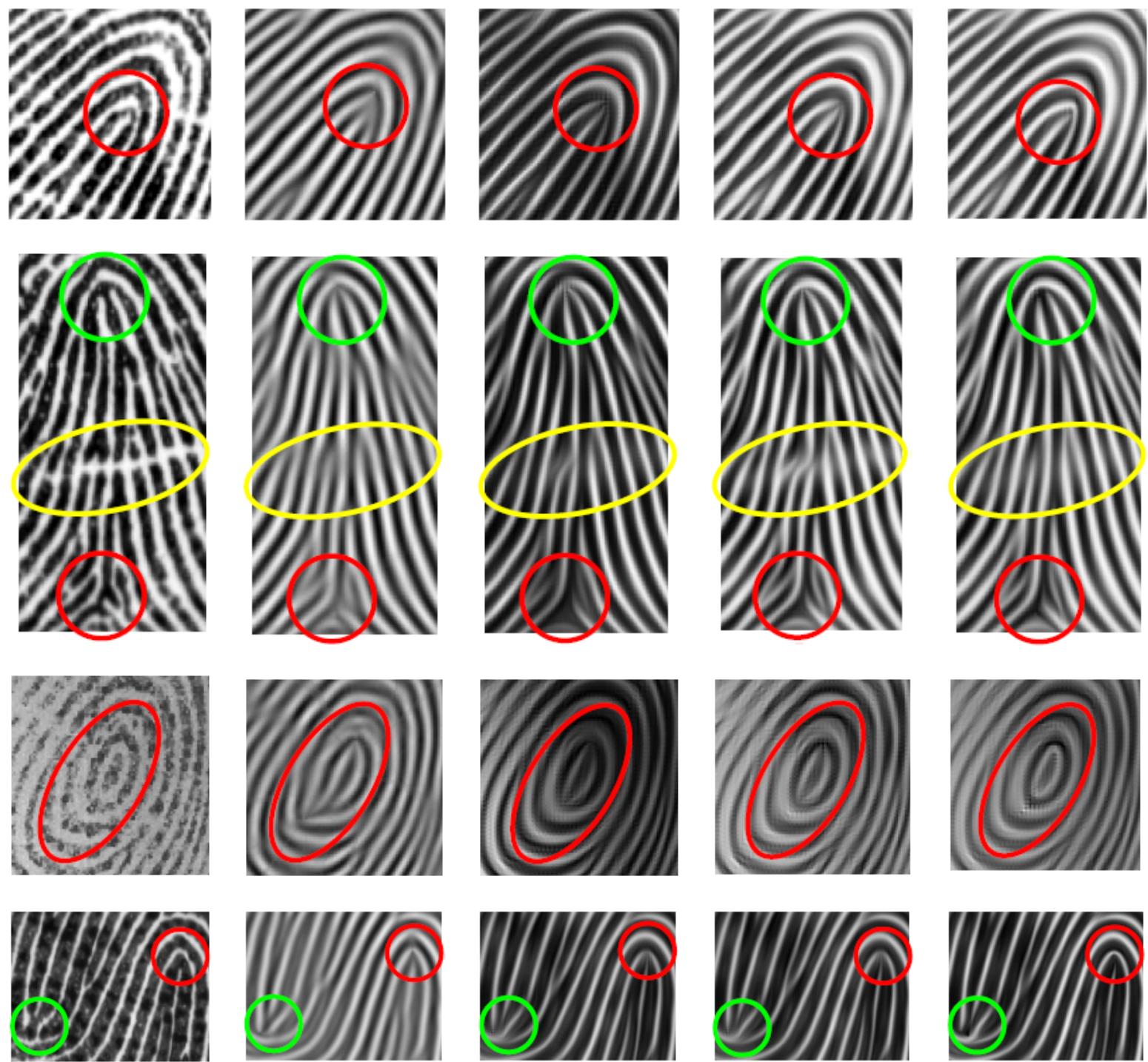

Figure 6. The enhancement results of Fig. 5. From the left-most column to the right-most column: part of the original images, and the corresponding results of the Gabor filter based method, Cheng's method, Hastings' method, and the proposed method.

Transaction on Pattern Recognition and Machine Intelligence, 20(8):777-789, 1998. 1, 5

[12] D. Maio, D. Maltoni, R. Cappelli, J. L. Wayman, and A. K. Jain. Fvc2002: Second fingerprint verification competition. ICPR2002, pages 811-814, 2002. 5

[13] D. Maltoni, D. Maio, A. K. Jain, and S. Prabhakar. Handbook of Fingerprint Recognition. Springer, New York, 2003. $1,3,5$

[14] P. Perona. Orientation diffusions. IEEE Transactions on Image Processing, 7(3):457-467, 1998. 2, 3, 4

[15] P. Perona and J. Malik. Scale-space and edge detection using anisotropic diffusion. IEEE Transactions on Pattern Analysis and Machine Intelligence, 12(7):629-639, 1990. 1, 2

[16] M. Proesmans, E. J. Pauwels, L. J. V. Gool, T. Moons, and A. Oosterlinck. Image enhancement using nonlinear diffu- sion. CVPR93, pages 680-681, 1993. 2

[17] A. R. Rao and R. C. Jain. Computerized flow field analysis: oriented texture fields. IEEE Transactions on Pattern Analysis and Machine Intelligence, 14(7):693-709, 1992. 1

[18] B. G. Sherlock, D. M. Monro, and K. Millard. Fingerprint enhancement by directional fourier filtering. IEE Proceedings Vision Image and Signal Processing, 141(2):87-94, 1994. 1

[19] J. Weickert. Anisotropic Diffusion in Image Processing. Teubner-Verlag, 1998. 2

[20] J. Weickert. Coherence-enhancing diffusion filtering. International Journal of Computer Vision, 31:111-127, 1999. 1, 2, 5

[21] M. Xie and Z. Wang. Fingerprint enhancement based on edge-directed diffusion. ICIG04, 2004. 2 